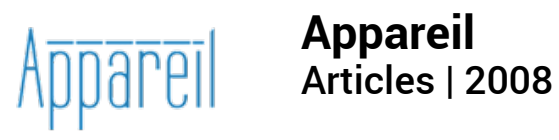

\title{
Notes sur le « on », le milieu et la vérité de l'appareil
}

Éric Méchoulan

\section{(2) OpenEdition}

Journals

Electronic version

URL: http://journals.openedition.org/appareil/124

DOI: 10.4000/appareil. 124

ISSN: 2101-0714

\section{Publisher}

MSH Paris Nord

\section{Electronic reference}

Éric Méchoulan, « Notes sur le « on », le milieu et la vérité de l'appareil », Appareil [Online], Articles, Online since 10 February 2008, connection on 30 July 2020. URL : http://journals.openedition.org/ appareil/124 ; DOI : https://doi.org/10.4000/appareil.124

This text was automatically generated on 30 July 2020 .

\section{(c) (i) (9)}

Appareil est mis à disposition selon les termes de la Licence Creative Commons Attribution - Pas d'Utilisation Commerciale - Pas de Modification 4.0 International. 


\title{
Notes sur le « on », le milieu et la vérité de l'appareil
}

\author{
Éric Méchoulan
}

1 La notion d'appareil mise de l'avant par Jean-Louis Déotte est souvent liée à la production des apparences ou à des formes d'apparaître, donc à des jeux de surface. Mais il faut d'entrée de jeu écarter une des habitudes intellectuelles les plus courantes en ce qui concerne ces jeux d'apparence et ce qui les sous-tendrait. La démarche de Jean-Louis Déotte opère en dehors de toute herméneutique qui aimerait trouver sous ces effets de surface les profondeurs, par définition, mystérieuses de la signification. Il exploite fréquemment les réflexions de Walter Benjamin, en tâchant manifestement d'en prolonger certaines intuitions. Il faut donc bien prendre pour lui-même ce qu'il souligne explicitement chez Benjamin: le fait qu'il «n'appartient donc pas au mouvement moderne de la subjectivité phénoménologique, à sa définition de la vérité, parce qu'il dissocie la conscience de la connaissance. C'est pour cette raison que l'interprétation (herméneutique) est sans valeur pour lui. [...] Ce qui nous conduira à dire que pour lui la photo et le cinéma sont des appareils de vérité plus que des dispositifs de perception ${ }^{1}$. " Ainsi, contrairement à ce que l'on pourrait penser, si l'appareil a affaire avec l'apparaître, ce n'est pas sous le signe phénoménologique de la perception ni sous celui, herméneutique, de l'interprétation. On sent ici une intuition productive plutôt que des formes de réception : l'appareil fait apparaître, il ne dispose pas simplement à recevoir ou même à enregistrer ${ }^{2}$.

Qu'est-ce qui est ainsi produit? Une médiation. On peut dire que l'appareil lie trois instances : le corps, la loi et l'événement. «L'appareil, c'est donc la médiation entre le corps (la sensibilité affectée) et la loi (la forme vide universelle)» (EA, p. 28); «Un appareil est un certain mode d'enchaînement légal sur l'événement.» (EA, p. 103) L'appareil ouvre le corps à autre chose que lui-même en inventant des formes de temporalité comme modes d'enchaînement des événements où se lient corps et loi. C'est en ce sens que l'on peut dire que le corps est pour la loi tout en étant aussi ouvert à l'événement. Loin d'être clos et circonscrit abusivement par l'ordre juridique, c'est par la loi que tout corps peut être ouvert à l'événement. 
3 En rendant «pareils » des corps (ce qui n'équivaut pas à les rendre tous identiques), l'appareil les ouvre à la forme universelle de la loi quand elle prend consistance dans un événement. Ce trio de termes est rarement conçu dans un enchaînement aussi strict que celui que propose Jean-Louis Déotte. Généralement le corps s'oppose ou résiste à la loi, qui en nie la singularité, ou la loi ne sait que faire des événements qui individualisent toujours plus des corps. Ici, au contraire, les trois éléments sont nécessaires pour que, de façon positive, l'appareil devienne productif.

On peut retrouver cette même puissance positive dans une autre caractéristique de l'appareil: le fait qu'il ne tourne pas à l'exercice d'une domination, mais qu'il ouvre plutôt sur une émancipation. Il est facile de discerner ici un contresens à ne pas faire : à la différence des « appareils idéologiques d'État » déterminés par Louis Althusser, il ne s'agit pas d'outils institués au service de l'État et de la classe dominante ${ }^{3}$. À côté des rapports de force et des moyens de répression (police, tribunaux, prisons, etc.), Althusser discernait des formations idéologiques qui prenaient corps, soutenaient et légitimaient les pouvoirs en place (école, famille, Église, médias d'information, institutions culturelles, etc.). Or, pour Jean-Louis Déotte, même si les appareils nécessitent un passage par l'imaginaire ${ }^{4}$, et même si certains d'entre eux sont bien des institutions culturelles d'État (les musées par exemple), il n'est pas question de ramener les appareils à un ordre étatique ou à une instance de domination. Au contraire, il en fait la marque même de l'émancipation : « la spécificité des appareils est de nous émanciper de l'adhésion originaire au corps et aux lieux. Les appareils suspendent, déracinent, arrachent, délocalisent, déplacent violemment les corps. » (EA, p. 52)

Quand bien même les appareils ne seraient pas des instruments de domination (de classe), ils n'en font pas moins usage de violence sur les corps. Dans nos sociétés occidentales, le premier de ces appareils (que ne mentionne pas Jean-Louis Déotte) est peut-être justement ce que les Romains appelaient mancipatio et emancipatio. Le droit de propriété ne venait pas au sens strict d'un achat, mais de la saisie immédiate par la manus dans une cérémonie rituelle: en présence des deux parties et de cinq témoins (des citoyens adultes) plus un porte-balance, l'« acquéreur » frappait la balance d'un lingot de métal en prononçant la formule suivante: «Hunc ego hominem meum esse aio ex jure quiritum isque mihi emtus esto hoc aere aeneaque libra» (Je dis que cet homme est à moi d'après le droit quiritain et je l'achèterai avec l'airain et la balance d'airain). Il faut, en effet, noter que la première proposition est au présent tandis que la seconde est au futur: si l'esclave est déjà sous sa possession, il n'y a pas de véritable achat. La main s'est abattue sur lui et l'autorité repose sur la force implicite de cette saisie. Appareil de possession ou de capture qui repose sur ce dédoublement temporel de la machinerie légale: le corps est ouvert à la loi par l'événement rituel de la mancipatio ${ }^{5}$. Parallèlement, l'emancipatio implique la libération de la manus par vente. L'enfant ou l'esclave qui sont sans droits, sous la pleine possession du pater familias doivent être vendu rituellement trois fois et ce sont ces ventes successives qui leur permettent de sortir de la puissance paternelle ${ }^{6}$.

6 Lorsque Jean-Louis Déotte affirme que, pour Benjamin, « la photo et le cinéma sont des appareils de vérité plus que des dispositifs de perception » (EA, 211-212), nous avons vu que nous sortions des références phénoménologiques et herméneutiques, nous évitons aussi un ancrage foucaldien tacitement présent dans la notion de "dispositif ». Pour Déotte, un dispositif, au mieux, prend les allures rhizomatiques du tableau ( la série 
des séries", comme le dit Michel Foucault) et forme alors une configuration à l'intérieur de l'épistémé, répondant au devoir d'efficacité et de gestion des informations. Cependant, l'événement ne peut jamais ouvrir le tableau, puisque celui-ci n'accueille le nouveau que sous la figure de la variation à l'intérieur de la série, - une tradition du nouveau. L'appareil, lui, doit ouvrir à l'événement.

On trouve dans une entrevue de Michel Foucault quelques éléments qui permettent de préciser ce qu'il entendait par dispositif: "un ensemble résolument hétérogène, comportant des discours, des institutions, des aménagements architecturaux, des décisions réglementaires, des lois, des mesures administratives, des énoncés scientifiques, des propositions philosophiques, bref : du dit, mais aussi du non-dit, voilà les éléments du dispositif. Le dispositif lui-même, c'est le réseau qu'on peut établir entre tous ces éléments ${ }^{7}$ ». Il indique, un peu plus loin, les rapports entre dispositif et épistémè : «C'est ça, le dispositif : des stratégies de rapports de force supportant des types de savoir, et supportés par eux. Dans Les mots et les choses, en voulant faire une histoire de l'épistémè, je restais dans une impasse. Maintenant, ce que je voudrais faire, c'est essayer de montrer que ce que j'appelle dispositif est un cas beaucoup plus général de l'épistémè. Ou plutôt, que l'épistémè, c'est un dispositif spécifiquement discursif, à la différence du dispositif qui est, lui, discursif et non-discursif, ses éléments étant beaucoup plus hétérogènes ${ }^{8}$.»

8 À l'instar des appareils, les dispositifs foucaldiens agissent par delà les épistémès repérables et sont constitués d'éléments hétérogènes. La grande différence tient une nouvelle fois à la question du pouvoir : un dispositif est une stratégie de rapport de forces, un appareil est une tactique d'émancipation des sujets. Le rapport de l'un à l'autre peut être double: soit un appareil surgit d'un dispositif par une procédure de retournement et de subversion, soit un appareil se dégrade en reproduction dans un dispositif.

9 Suivant Walter Benjamin, Jean-Louis Déotte prend l'exemple du cinéma: d'abord dispositif de surveillance, le cinéma devient appareil à partir du moment où il repose sur le montage, soit chez Eisenstein et Vertov: «le cinéma est devenu un appareil artistique à part entière du fait de l'adoption d'une écriture : le montage. Car l'écriture par montage, comme le montre Kracauer, non seulement respecte les conditions de la production industrielle (discontinuité radicale des moments et des lieux de tournage par rapport à la fiction narrative et à la continuité spatiale des décors de la diégèse), mais surtout rompt avec toute emprise fantasmagorique. La discontinuité assumée des plans-séquences vient casser toute séduction rêveuse du pouvoir des images. » (EA, p. 106). Le montage subvertirait donc le dispositif de production de la fantasmagorie. Cependant, «cet arrachement n'est jamais définitif, la continuité de la fantasmagorie reprend toujours le dessus, les appareils se mettent à réalimenter, selon leur régime propre, la rêverie de l'intérieur » ( $E A$, p. 106), autrement dit, le dispositif récupère la puissance d'un appareil pour lui faire produire du continu, alors qu'un appareil faisait apparaître justement parce qu'il interrompait.

Cet exemple est significatif de la place allouée à l'art. En effet, même si les appareils donnés en exemple ne concernent pas tous a priori le domaine artistique ("pour la modernité : la perspective, la camera obscura, le musée, la photographie, le passage urbain, le cinéma, la cure analytique, etc. », EA, p. 27), et même si « la situation d'un art à un moment quelconque n'est intelligible que si l'on ne confond pas tel art et tel appareil, par exemple la peinture et la perspective » (EA, p. 53), il n'en demeure pas 
moins qu'il semble que la vertu de l'interruption, de la subversion, de la discontinuité soit bien une effectivité des œuvres d'art: « Pour lutter contre ce mouvement naturel [la dégradation de l'appareil en dispositif], il faudra des œuvres jamais vues ou entendues, qui, pour chaque appareil, en allant plus loin que els œuvres précédentes, rappelleront tel ou tel enjeu d'époqualité.» (EA, p. 106) Les œuvres d'art doivent ainsi se succéder en introduisant à chaque fois une discontinuité fondamentale en résonance avec l'appareil dont elles retrouvent et déploient à chaque fois la puissance propre d'ouverture des corps à la loi : «On pourra donc dire de l'œuvre d'un art appareillé qu'elle devra introduire une discontinuité pour être au plus près de la spécificité temporelle de cet appareil'^.» (EA, p. 57)

11 Il s'agit là, en effet, d'un point crucial pour bien saisir ce que produit un appareil à la différence du dispositif (spatialisé dans le tableau): essentiellement, de nouvelles formes de temporalité, quelque chose à quoi nous ne sommes pas par avance disposés. "Les appareils ne s'affrontent pas sur le terrain "ontologique", au sens où certains seraient plus réalistes que d'autres, mais sur celui de l'émancipation et de la complexification des inventions de temporalité » $(E A$, p. 52) C'est pourquoi la question de la discontinuité a autant d'importance, parce qu'elle implique de rompre avec une conception de la temporalité en en proposant une autre expérience. L'appareil pose à chaque fois le problème de sa propre "époqualité »: comment faire époque? Autrement dit, comment organiser la venue (toujours surprenante et inattendue) de l'événement? (d'où le titre même de l'ouvrage : « l'époque des appareils »).

Il faut, bien sûr, différencier une époque et une épistémè: l'appareil n'est ni disciplinaire (à tous les sens du terme) ni dispositif général de contrôle ou de surveillance. L'appareil configure une époque en y installant des formes inédites de temporalité inscrites sur des supports et des mediums et permettant de médiatiser événement, corps et loi. Il constitue donc à chaque fois des milieux. D'où l'intérêt, avec le premier chapitre, de partir du cas de Schiller pour lequel « la culture est le milieu de l'art et de la politique » (EA, p. 9-28). Mais il faut aussi entendre milieu comme ce qui est " entre » et interroger alors tout médium à partir de cet « entre » et du milieu qu'il fabrique.

Prenons l'exemple de Descartes auquel Jean-Louis Déotte consacre un de ses chapitres. Il avait déjà fait remarquer que « l'appareil qui constitue le socle de la modernité - la perspective - a émancipé les « sujets » de la métaphysique du récit chrétien de la Faute et de la Rédemption" ( $E A$, p. 56) et que la perspective "consiste bien sûr en une rationalisation de l'espace, mais surtout, elle a fait surgir un nouveau mode de la temporalité en rabattant le temps sur l'espace: l'instant comme découpe dans un continuum homogène de temps, et une nouvelle détermination de la singularité : l'ego cogito.» (EA, p. 51) Descartes ne constitue donc pas un exemple anodin, il figure comme l'arkhé (ou l'archive) philosophique du moderne, justement parce que le point du temps du cogito « n'est rien sans la présence de l'archive, de l'infini comme archive. Appelons cette idée non pas idée de Dieu, mais loi, archive de la loi, arkhé au sens de commencement, origine, fondation, primauté. Le cogito consiste alors à s'alimenter de la certitude d'être pour la loi, du fait de l'avoir été au moins une fois pour un instant. Du fait d'une loi qui n'est pas celle de l'instant. » (EA, p. 141).

De fait cette inscription dans le temps est cruciale puisqu'elle donne accès à l'idée claire et distincte de mon existence comme chose pensante, mais seulement dans le moment de la performance orale ou mentale. La question que ne peut manquer de poser 
Descartes est alors: «je suis, j'existe, cela est certain; mais combien de temps [quandiu] ? à savoir autant de temps [Nempe] que je pense ${ }^{10} »$. Il faudrait donc que le sujet cartésien répète et répète encore, à chaque instant, l'opération de l'énonciation pour qu'il soit assuré de traverser le temps d'un pas débonnaire. Cependant, il me faut une garantie que cette même pensée-existence se conserve bien dans la durée vacillante des jours. Cet être différent qui me conserve dans la durée de l'existence n'est pas simplement «ce que j'appelle Dieu », mais est Dieu. Dieu est le garant, hors langage, du temps, son indispensable archive, mais à condition qu'en lui se conjoignent ce qui me permet à la fois de durer et de sortir du langage.

Descartes se méfie, en effet, de la place prise par le langage dans le mouvement de la pensée. Il souligne, sans doute, l'aspect linguistique nécessaire par où le cogito peut apparaître, mais l'équivalence entre l'énonciation verbale et la conception spirituellene suppose pas une impeccable superposition, car, même dans l'esprit les mots se glissent, le langage interfère, les faux amis se bousculent. À la fin de son examen du morceau de cire, Descartes ne bute pas seulement sur l'obstacle des sens, mais aussi sur l'opacité du langage : «Car encore que sans parler [tacitus \& sine voce, silencieux et sans parole] je considère tout cela en moi-même, les paroles toutefois m'arrêtent, et je suis presque trompé par les termes du langage ordinaire [hœreo tamen in verbis ipsis, \& fere decipior ab ipso usu loquendi, je suis toutefois arrêté par les mots, et je suis presque abusé par l'usage même du langage] » (Méditation II). C'est bien l'usage qui égare et qui trompe (en particulier, dans le cas du morceau de cire, par le mouvement du discours qui replie trop vite la possibilité de voir sur la nécessité de juger). Le langage se trouve là où il ne devrait pas opérer, empiétant sur la seule puissance de penser. Plus encore, le langage se trouve au point de fondement, alors qu'on devrait y découvrir la pensée hors de l'emprise des mots. Descartes reporte alors sur le vulgaire ces mauvaises manières de parler : «Un homme qui tâche d'élever sa connaissance au delà du commun [vulgus] doit avoir honte de tirer des occasions de douter des formes et des termes de parler du vulgaire. "(Méditation II). Conformément aux tenants du purisme malherbien, le langage est aussi un signe de distinction sociale; les mauvais usages de la parole sont seuls en cause. Plus profondément, cependant, on voit la position fondamentale qu'en vient à occuper le langage, jusqu'à déployer ses manières dans le silence même de la pensée.

L'idée claire et distincte de l'existence doit donc déborder d'elle-même dans le temps pour accéder à l'existence de Dieu et doit résorber en elle ce qui glisse constamment des mots sous les idées. L'immédiateté de la communication angélique n'est atteignable en pensée qu'à condition de prendre ses distances avec la communication rugueuse du vulgaire. Or, Descartes est loin d'intérioriser simplement le modèle de la communication angélique, il loge aussi sa pensée dans les plis de nouveaux rapports à la langue et à l'éloquence et de nouveaux façonnages des figures d'auteur.

On assiste, dans les premières décennies du XVIIe siècle, à une certaine "délocalisation" de l'éloquence qui passe d'un lieu savant à des usages littéraires socialement plus variés et à une "dislocation » de la rhétorique ${ }^{11}$, dans la mesure où des trois catégories principales (l'invention, qui ouvrait le trésor ancien des idées, des principes et des arguments; la disposition, qui en réglait l'articulation logique; l'élocution, qui en façonnait les tournures singulières), la première, qui était la plus importante puisqu'elle permettait au nouveau de se nouer aux légitimités de l'ancien, 
apparaît désormais comme secondaire par rapport à la disposition qui en ordonne méthodiquement les apparitions et par rapport à l'élocution qui en enrobe les leçons.

Descartes compte parmi ses amis Jean-Louis Guez de Balzac qui, dans les années 1620, a secoué le monde des lettres, en faisant de lui-même comme auteur le foyer explicite et unique de l'éloquence. Ses adversaires le traitaient d'insupportable vaniteux, de flatteur indécent ou de girouette ignorante, mais Descartes n'hésita pas à louer le style de son $a^{2} i^{12}$. Reste le problème de savoir ce qui peut assurer Balzac de sa propre rareté : de qui tient-il le pouvoir de distribuer ainsi la gloire? Qui lui a donné souveraineté sur le royaume de l'éloquence ? Personne d'autre que lui-même (et le clan d'amis et d'alliés qu'il a su constituer autour de lui). Il y a bien une forme d'autoposition de l'auteur Balzac que l'on va retrouver dans un autre épisode encore plus célèbre: la querelle du Cid. Ce qui envenime les relations entre Corneille et ses confrères, c'est un vers dans une épître qu'il fait circuler après le succès de la pièce en 1637: "Je ne dois qu'à moi seul toute ma renommée ». La querelle va déborder rapidement cet élément, mais il est caractéristique que Corneille tente, comme Balzac avant lui, de ne plus reconnaître les dettes, les héritages ou les reconnaissances envers les auteurs anciens, ses confrères actuels, voire ses patrons. L'auteur devient un foyer de discours, indépendant de toute autorité qui lui serait extérieure, de même que le Descartes qui se met en scène dans le Discours de la méthode cherche dans la mise à l'écart de toutes les formes d'autorité l'ordre nécessaire pour que l'évidence claire et distincte du point fixe du cogito apparaisse. La revendication de clarté n'a de sens qu'en vertu de cette auto-position du sujet : dans la figure de l'auteur se nouent la production immédiate de soi sur la scène publique et la transparence à soi lorsque toutes les formes d'autorité extérieures ont été évacuées.

19 Toutes formes d'autorité extérieure ? C'est en fait peu probable. Et c'est bien là où la question de l'appareil constitue une manière de reprendre à un autre niveau le propos cartésien. "Cette passibilité du je, le fait d'être redevable d'une loi déjà là et pour toujours, en quelque sorte en attente depuis toujours d'un sujet archiviste, c'est, d'une part, l'assurance d'une permanence au-delà du point temporel et, d'autre part, la certitude de ne pas être nécessairement livré aux créances langagières et aux fantasmagories de l'incarnation. [...] car cette passibilité doit être réassurée : instituée et appareillée. L'écriture est la première institution et il n'y a pas d'institution sans écriture.» (EA, p. 141)

20 Il faut bien, en effet, faire la part d'une posture, d'une mise en scène admissible, reconnaissable, valorisante, et des nécessités de la publication : par en haut, grâce aux institutions du sens, par en bas, au moyen des supports de la communication. Descartes en a été fort conscient: loin de se ramener à ce trait sans épaisseur du cogito, il en déplie rigoureusement les implications publiques et construit avec soin ses stratégies d'auteur.

21 Il faut, d'abord, un privilège. Depuis 1566, c'est obligatoire, mais c'est surtout à partir des années 1620 que la Grande Chancellerie en délivre systématiquement: ils sont alloués généralement pour cinq ans et vont parfois jusqu'à dix ans. Dans trois quarts des cas, en 1636, ils sont alloués aux libraires et non aux auteurs. Grâce aux services de Mersenne qui a des entrées à la Grande Chancellerie, Descartes obtient un privilège de dix ans à son nom et non seulement pour cet ouvrage, mais pour ceux à venir également, ce qui est tout à fait exceptionnel. La stratégie de communication de l'auteur commence donc bien avec les reconnaissances institutionnelles et la publicité 
qui entoure la publication. Parallèlement au privilège qui concerne le commerce du livre, la censure fait rapport sur le contenu de l'ouvrage. Descartes a toujours été extrêmement prudent avec les censures ecclésiastique et royale, au point de déclarer en avoir, en fait, intériorisé les principes : les censeurs sont moins « rigoureux » qu'il ne l'est pour lui-même (Méditation VI). Le sujet cartésien qui semble se retirer dans l'abstraction intellectuelle d'un point d'énonciation n'y parvient qu'à condition d'en organiser soigneusement la réception publique jusqu'en soi-même ${ }^{13}$. Par ailleurs, il ne faut pas oublier que les premières publications des Méditations en 1641et 1642 ne comportaient pas, comme aujourd'hui, le seul texte des six méditations : après les 89 pages des méditations à proprement parler suivaient 258 pages d'objections faites par divers personnages et 377 pages de réponses. Le public était bien présent matériellement dans l'espace du livre.

C'est aussi ce que son attention aux supports même de ses publications indique. Il avait l'habitude de demander aux libraires l'impression d'une ou deux dizaines d'exemplaires avec des marges plus grandes qu'à l'habitude afin d'en faire don à ses amis et connaissances. Les grandes marges devaient leur permettre d'écrire leurs propres commentaires tout à leur aise, comme s'il cherchait à allouer à la communication de ses écrits le tour aristocratique d'une conversation de salon. Alors que tous les livres sont imprimés sous forme continue sans paragraphes distincts, le Discours de la méthode est le premier texte (après l'exemple du Prince de son ami Guez de Balzac publié en 1631) à reprendre la méthode de l'alinéa et de la numérotation en marge (seule la Bible était ainsi publiée depuis le XVIe siècle ${ }^{14}$ ). Descartes en imposant un découpage analogue à celui de la Bible joue à deux niveaux : il hausse son texte au statut d'œuvre hors du commun, il se rend plus facilement accessible au public non savant qui va y trouver ainsi plus de "clarté ». Il a donc bien conscience d'une structure d'énonciation mise en valeur par les dispositifs matériels du support: la disposition rhétorique n'est pas uniquement une affaire de méthode, mais aussi d'imprimerie.

Ce sont là les moyens d'appareiller l'expérience du cogito dans un milieu qu'il peut projeter à partir de cette infime pointe de l'instant spéculatif, apparemment totalement isolé de son temps et du temps lui-même. Descartes apparaît ici bien exemplaire de cet appareillage de la modernité. On peut même dire qu'il en constitue un des «appareils de vérité » (comme cela a été dit du cinéma). Autrement dit, il n'en énonce pas la vérité, il en ordonne les configurations nécessaires pour que la question de la vérité lui soit intimement liée. C'est dire que l'on retrouve ici un propos foucaldien (au sens où l'interrogation de Michel Foucault ne portait pas sur la folie ou sur la sexualité comme telle, mais sur le fait surprenant que les discours sur la folie ou sur la sexualité aient liés étroitement ces objets à une vérité sur l'homme).

24 C'est peut-être en cela qu'un autre point commun demeure entre le dispositif foucaldien et l'appareil déottien : leur surgissement impersonnel. Malgré la référence à la stratégie, le dispositif ne provient pas d'une rationalisation homogène d'individus (voire d'une "classe dominante »), mais de formes hétérogènes qui s'appuient ou se contestent individuellement sans plan d'ensemble. De même, l'appareil est causa sui : "C'est une affaire d'origine et non de commencement. Parce qu'à partir de cette révolution (et de ce point de vue, on peut dire que l'humanité aura connu peu de révolutions: celles des appareils), c'est le mode d'accueil de l'événement qui change. L'événement est par définition imprévisible. » (EA, p. 111) Le modèle demeure ici celui de l'œuvre d'art, par définition, inédite et imprévue qui crée son propre public en 
inventant, en quelque sorte, la configuration de sa réception: «L'énigme qui tire en avant les œuvres d'art comme un trou d'air à la proue d'un mobile qui l'aspire, c'est celle de la décision, que personne n'a pu prendre, de l'avènement de tel appareil ou de tel genre littéraire. L'énigme donc de l'origine de la circonscription de tel apparaître, de telle spatialité, de telle temporalité. » (EA, p. 112)

Tel est peut-être ce qui conduit l'auteur lui-même à des procédures stylistiques de l'impersonnel. Par exemple: "Dès lors, on ne se demandera pas en quoi [...]. On insistera au contraire sur $[. .] ».(E A$, p. 73) ou « On retrouve la même démarche chez Marx quand [...]. On insistera donc sur [...] » (EA, p. 76). Il y a là un exercice du « on » qui trouve toute sa valeur lorsque ce pronom impersonnel devient le sujet même de l'époque appareillée: "l'irruption imprévisible de l'appareil comme ce qui a fait époque, le moment d'autoconstitution de l'appareil qui n'est pas celui de l'invention technique; l'instant où il est devenu évident pour un certain public que, du fait de tel appareil, "on" changeait d'époque.» (EA, p. 111) D'un «on» à l'autre, on peut s'interroger sur le redoublement ou la différence. Qui se glisse sous ce pronom impersonnel?

D'autant que la place même de la philosophie (donc du philosophe) apparaît très classiquement centrée. Nous avons vu l'enjeu des œuvres d'art à l'intérieur des appareils. Or, il est très notable que se joue là un rapport à la vérité qui prolonge l'indirection allouée à l'art et le caractère direct du discours philosophique, seul susceptible de dire vraiment ce qu'il en est de la vérité : "L'œuvre a donc affaire, à sa manière, indirecte, à la vérité, parce qu'un appareil, à la différence d'un dispositif, comporte une vérité. Mais si l'œuvre d'un appareil comporte une vérité, il revient à la seule philosophie de l'exposer. » (EA, p. 131)

Dire qu'un appareil « comporte » une vérité n'est pas identique au fait de souligner que des appareils sont des appareils de vérité. Par ailleurs, qu'est-ce qui donne à la philosophie cette situation particulière, ce surplomb spécifique, qui semble résider en dehors des appareils ? Et l'exposition en question participe-t-elle de la «mise en forme de l'apparence par des appareils culturels, comme le musée et l'exposition » (EA, p. 16) ? Autant de questions sur l'appareillage du discours philosophique lui-même.

\section{NOTES}

1. Jean-Louis Déotte, L'époque des appareils, Paris, Éditions Lignes \& Manifestes, 2004, p. 211-212. Désormais j'inclurai les références directement dans le corps du texte après l'abréviation « EA ».

2. «Paradoxalement donc, l'appareil est davantage une technique de suspension et d'oubli que d'enregistrement du visible. »EA, p. 96-97.

3. Louis Althusser, "Idéologie et appareils idéologiques d'État ", dans Positions, Paris, éditions sociales, 1982 [1970].

4. «Les appareils doivent extraire leur matériau non du réel sensible mais du plasma imaginal » (EA, p. 51). 
5. Voir R. von Jhering, L'esprit du droit romain dans les diverses phases de son développement, trad. 0 . de Meulenaere, Paris, A. Maresq, 1886, p. 107-151; Paul Huvelin, Cours élémentaire de droit romain, Paris, Sirey, 1927, t. I, p. 430-431 et 485-497.

6. Les enfants sont "vendus" à un créancier et affranchi par lui, or une fois affranchi ils reviennent automatiquement sous la manus paternelle. Mais si la vente a lieu trois fois, au troisième affranchissement, ils ne peuvent plus revenir sous l'autorité du père et sont donc libres. Voir Léopold Thézard, Répétitions écrites sur le droit romain, Paris, Ernest Thorin, 1885, p. 57-58.

7. Michel Foucault, «Le jeu de Michel Foucault », Dits et écrits, III, 1976-1979, Paris, Gallimard, 1994, p. 299.

8. Michel Foucault, « Le jeu de Michel Foucault », Dits et écrits, III, 1976-1979, p. 300-301.

9. On peut trouver chez Giorgio Agamben une autre tentative pour déplacer les dispositifs : la profanation qui rend à l'usage commun ce qui en avait été séparé. Voir Qu'est-ce qu'un dispositif?, trad. Martin Rueff, Paris, Rivages, 2007.

10. René Descartes, Meditationes de prima philosophia: Méditations métaphysiques, trad. de Luynes, intro. et notes de Geneviève Rodis-Lewis, Paris, Vrin, 1970. Je développe ce cas de Descartes dans un ouvrage collectif à paraître, dirigé justement par Jean-Louis Déotte, Le milieu des appareils.

11. Voir Hélène Merlin, Public et littérature en France au XVIIe siècle, Paris, Les Belles Lettres, 1994, ainsi que Christian Jouhaud, Les pouvoirs de la littérature: Histoire d'un paradoxe, Paris, Gallimard, 2000.

12. L'épître latine est donnée dans la Correspondance éditée par Adam et Tannery. La traduction française se trouve dans l'édition des Lettres faite par Clerselier en 1663 et reprise dans l'édition de 1667 : Lettres de Mr Descartes. Où sont traitées plusieurs belles Questions Touchant la Morale, Physique, Médecine \& les Mathematiques, Paris, Charles Angot, 1667, p. 470-471.

13. Voir Jean-Pierre Cavaillé, «"Le plus éloquent philosophe des derniers temps" : les stratégies d'auteur de René Descartes ", Annales HSS, no 2, mars-avril 1994, p. 349-367 ; ainsi que Stéphane Van Damme, Descartes : essai d'une histoire culturelle d'une grandeur philosophique, Paris, Presses de sciences po, 2002.

14. Henri-Jean Martin, La naissance du livre moderne (XIVe-XVIIe siècles). Mise en page et mise en texte du livre français, Paris, Éditions du Cercle de la Librairie, p. 319-327. 